\title{
Paletli Bir Mobil Robotun Modellenmesi ve Bulanık Mantık ile Yörünge Kontrolü
}

\author{
Osman DOĞMUŞ ${ }^{* 1}$, Mahit GÜNEŞ ${ }^{1}$ \\ ${ }^{1}$ Kahramanmaraș Sütçü Imam Üniversitesi, Mühendislik Mimarlık Fakültesi, Elektrik \\ Elektronik Mühendisliği Bölümü, Kahramanmaraş
}

Gelis tarihi: 23.03.2020

Kabul tarihi: 30.07 .2020

\section{Öz}

$\mathrm{Bu}$ çalışmada, paletli otonom bir araç tasarlanmış olup tasarlanan otonom aracın yörünge kontrolü bulanık mantık denetleyici (BMD) kullanılarak gerçekleştirilmiştir. Çok amaçlı tasarlanan otonom araç, otomatik yönlendirme, belirli bir görevi icra etme, çeşitli ortamlara yük taşıyabilme özelliklerine sahiptir. Otonom araçların yörünge kontrolünde farklı kontrol teknikleri kullanılmaktadır. Otonom robotun yörünge kontrol yapısının performansını klasik PI denetleyici ile karşılaştııılmış ve kontrol sistemi Matlab/Simulink modeli kullanılarak benzetim çalışmaları gerçekleştirilmiştir. BMD’nin sistemin performansını artırdığı ve daha kararlı bir yapı sağladığı gerçekleştirilen benzetim çalışmaları ile ortaya konulmuştur.

Anahtar Kelimeler: Paletli mobil araç, Dinamik modelleme, Kinematik modelleme, PI, BMD

\section{Modeling a Tracked Mobile Robot and Trajectory Control by Using Fuzzy Logic}

\begin{abstract}
In this study, a tracked autonomous vehicle was designed and the trajectory control of the designed autonomous vehicle has been carried out using a fuzzy logic controller (FLC). The multi-purpose autonomous vehicle has the features of automatic steering, performing a specific task, carrying loads to various environments. Different control techniques are used in the trajectory control of autonomous vehicles. The performance of the orbital control structure of the autonomous robot has been compared with the classical PI controller and simulation studies were realized by using the control system Matlab/Simulink model. It has been demonstrated by simulation studies that BMD increases the performance of the system and provides a more stable structure.
\end{abstract}

Keywords: Tracked mobile robot, Dynamic modeling, Kinematic modeling, PI, FLC

\footnotetext{
*Sorumlu yazar (Corresponding author): Osman DOĞMUŞ, odogmus@ksu.edu.tr
} 


\section{GíRİş}

Paletli otonom araçlar yüksek manevra kabiliyetlerinin yanı sıra zor arazi şartlarında hareket edebilen, insanlar için tehlikeli olabilecek görevleri yerine getiren ve uzaktan kontrol edilebilen araçlardır. Bu araçlar arama kurtarma, bomba imha benzeri uygulamalar için kullanılmaktadır [1]. Bu çalışmada paletli otonom bir aracın kontrol yöntemlerini geliştirmek amaciyla bilgisayar ortamında modellemesi yapılmıştır.

$\mathrm{Bu}$ çalışmada paletli bir aracın matematiksel modeli çıkartılmış ve benzetim çalışmaları yapmak amaciyla Matlab/Simulink ortamında blok modelleri oluşturulmuştur. Günümüzde, PID kontrolörleri mobil robotların motor kontrolünde ve genel olarak endüstriyel kontrol sistemlerinde hala yaygın olarak kullanılmaktadır. Bununla birlikte, PID denetleyicilerin sabit parametrelere sahip olması nedeniyle doğrusal olmayan ve zamanla değișen parametreler içeren sistemlerin denetiminde düşük performans göstermektedir $[2,3]$. PID denetleyiciler hız, tork ve konum denetiminde motorun karmaşı bir matematiksel modeline ihtiyaç duyarlar. Son zamanlarda geleneksel denetim yaklaşımlarının istenmeyen özelliklerinden kaçınmak için yapay zekâ temelli bulanık mantık, yapay sinir ağları, sinirsel-bulanık mantık yöntemlerini kullanan denetleyici yapıları geliştirilmektedir [4,5]. Bulanık mantık denetleyicinin (BMD) yapısı uyarlanabilir özelliklere ve geniş çalışma koşullarına sahiptir. Ayrıca belirsizliklere, değişken parametrelere ve değişken yük dağılımına sahip sistemlerde kullanıldığında sistemin güçlü cevaplar vermesini sağlar [6]. Yüksek performans gerektiren uygulamalarda geleneksel PID denetleyici yerine BMD yaygın olarak kullanılmaktadır.

Bu çalışmada paletli mobil robotun denetimi için PI tipi denetleyici ve BMD tasarlanmıştır. Önerilen denetleyicinin başarısını kanıtlamak için aracın, referans olarak verilen sinüzoidal bir yörüngeyi izlemesi ve bozucu girişe karşı tepkisi incelenmiştir. Elde edilen sonuçlar PI tipi denetleyiciyle karşılaștırılmıștır.

\section{2. ÖNCEKİ ÇALIŞMALAR}

Sidi ve arkadaşları [7], üç serbestlik derecesine (3DOF) sahip paletli bir mobil robotun modellenmesini sunmuş ve Matlab/Simulink ortamında paletli hızını PID ile kontrol etmiştir. Model, paletli mobil robotun uzunlamasına, yanal ve sapma hareketlerindeki yerel koordinat davranışlarının yanı sıra araç hızı, palet kuvveti ve palet kayması gibi kinematik ve kinetik tepkilerini incelemek için geliştirilmiştir.

Huang ve arkadaşları [8], diferansiyel tahrikli paletli robot için ileriye dönük nokta bilgisine dayanan bir PI tipi yörünge izleme denetleyici geliştirmişlerdir. Global Navigasyon Uydu Sistemi antenleri ve bir alıcı modül ile robotun konum ve yön hatalarının gerçek zamanlı tahminlerini elde etmek için navigasyon sensörleri kullanılmıştır.

Asai ve arkadaşları [9], paletli mobil robotun bir yörüngeyi izleyebilmesi için PD benzeri yapay sinir ağı denetleyici yapısı kullanmışlardır. Önerilen çevrimiçi sinir ağ 1 denetleyicisi; kendini eğitebilme ve PD denetim benzeri geribildirim yapısı özelliklerine sahiptir.

Ji ve arkadaşları [10], iki DA motor tarafından sürülen paletli mobil robotta, yük bozulması ve sol palet ile sağ palet arasındaki mekanik yapı farkı gibi faktörlerin yörünge izleme hatasına neden olduğunu ve izleme hassasiyetini etkilediğini belirtmişlerdir. Bu sorunlara yönelik olarak, kayan bulut-model çapraz kuplaj denetleyici önermişlerdir. Hiz döngüsündeki kayan mod denetleyici, rastgele parazitleri engellemek ve tek motorun hız izleme hatasını azaltmak için tasarlanmıştır. Bulut-model çapraz kuplaj denetleyici ise her iki motorun hız izleme hatasını azaltmak için tasarlanmıştır.

\section{PALETLİ MOBÍL ARAÇ TASARIMI}

\subsection{Fiziksel Özellikler}

Tasarlanan aracın mekanik aksamlarının yapımı için; 2 adet $120 \mathrm{~W}$ DA motor, motor ve teker milleri için rulmanlı yataklar, 45 x $65 \mathrm{~cm}$ ebadında 
$8 \mathrm{~mm}$ alüminyum levha, çelik ve alüminyum dişliler ile 2 adet çift yönlü $1440 \mathrm{~mm}$ triger kayış kullanılmıştır. Şekil 1'de aracın Solid Works'de çizilmiş üç boyutlu modellemesi görülmektedir. Şekil 2'de ise yapılan paletli aracin resmi verilmiştir.

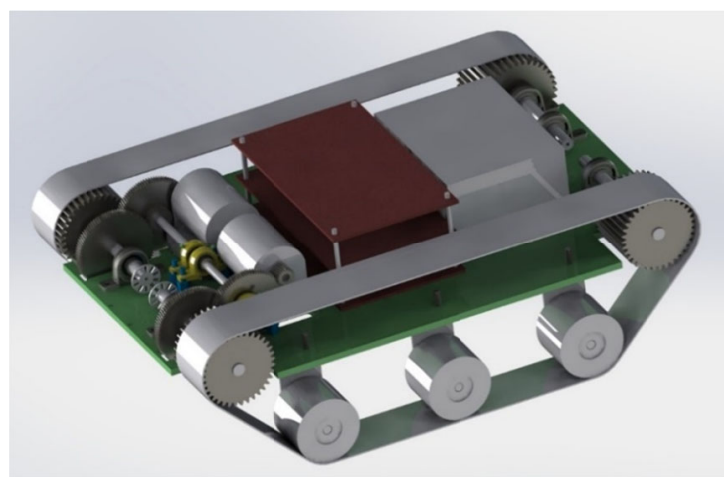

Şekil 1. Paletli aracın üç boyutlu modeli

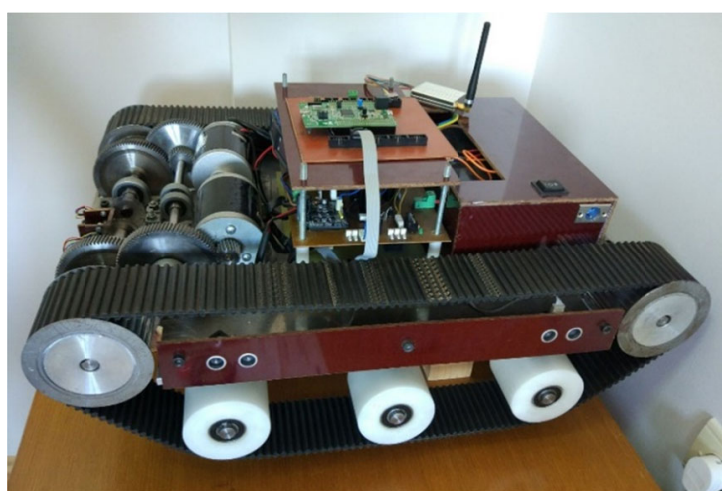

Şekil 2. Paletli aracın genel görünüşü

Çizelge 1. Paletli aracın fiziksel değerleri

\begin{tabular}{|l|c|c|c|}
\hline Parametre Adı & Simge & Birim & Değeri \\
\hline Kütle & $\mathrm{m}$ & $\mathrm{kg}$ & 36,37 \\
\hline En & $\mathrm{d}$ & $\mathrm{cm}$ & 45 \\
\hline Boy & $\ell$ & $\mathrm{cm}$ & 65 \\
\hline Palet çarkı yarıçapı & $r$ & $\mathrm{~mm}$ & 45,8 \\
\hline Dişli aktarma oranı & $\mathrm{N}_{\mathrm{g}}$ & - & 14,78 \\
\hline Hız & $v$ & $\mathrm{~m} / \mathrm{s}$ & 1,15 \\
\hline $\begin{array}{l}\text { Kütle Eylemsizlik } \\
\text { momenti }\end{array}$ & $\mathrm{J}$ & $\mathrm{kgm}^{2}$ & 1,875 \\
\hline
\end{tabular}

Çizelge 1'de paletli aracın fiziksel değerleri verilmiştir. Çizelge 2'de ise paletli araçta kullanılan DA motor parametreleri verilmiştir. Parametrelerin bir kısmı (L, R, $\left.\mathrm{K}_{\mathrm{b}}, \mathrm{K}_{\mathrm{T}}, \mathrm{b}\right)$ gerekli motor deneyleri yapılarak belirlenmiştir. Eylemsizlik momenti (J) hesaplanarak bulunmuştur [11].

Çizelge 2. DA motorun parametreleri

\begin{tabular}{|l|c|c|c|}
\hline Parametre Adı & Simge & Birim & Değeri \\
\hline Güç & $\mathrm{P}$ & $\mathrm{W}$ & 120 \\
\hline Devir & $\mathrm{n}$ & $\mathrm{d} / \mathrm{d}$ & 2750 \\
\hline Tork & $\mathrm{T}_{\mathrm{m}}$ & $\mathrm{Nm}$ & 0,42 \\
\hline Gerilim & $\mathrm{V}$ & $\mathrm{V}$ & 24 \\
\hline Akım & $\mathrm{I}$ & $\mathrm{A}$ & 5 \\
\hline Endüktans & $\mathrm{L}$ & $\mathrm{mH}$ & 1,59 \\
\hline Direnç & $\mathrm{R}$ & $\mathrm{Ohm}$ & 1,137 \\
\hline Ters EMK sabiti & $\mathrm{K}_{\mathrm{b}}$ & $\mathrm{Nm} / \mathrm{A}$ & 0,06467 \\
\hline Tork sabiti & $\mathrm{K}_{\mathrm{T}}$ & $\mathrm{Nm} / \mathrm{A}$ & 0,06467 \\
\hline $\begin{array}{l}\text { Viskoz Sürtünme } \\
\text { katsayısı }\end{array}$ & $\mathrm{b}$ & $\mathrm{Nm} / \mathrm{s}$ & $98,8.10^{-6}$ \\
\hline $\begin{array}{l}\text { Kütle Eylemsizlik } \\
\text { momenti }\end{array}$ & $\mathrm{J}$ & $\mathrm{kgm}$ & $1,38.10^{-6}$ \\
\hline
\end{tabular}

Matlab/Simulink ortamında araç modeli oluşturmak için Eşitlik 1-28' de verilen aracın kinematik ve dinamik formülleri kullanılmıştır. Araç modelinde kullanılan parametreler Çizelge 1 ve 2 'de verilmiştir.

\subsection{Paletli Aracın Matematiksel Modeli}

\subsubsection{Kinematik Model}

Kinematik model çıkarabilmek için paletli aracın koordinat düzlemindeki görünüşü Şekil 3'de gösterilmiştir. Matematiksel ifadeler ise Eşitlik 1-8'de verilmiştir [7,12].

$$
\begin{aligned}
& \mathrm{v}_{\mathrm{R}}=\mathrm{rw}_{\mathrm{R}} \\
& \mathrm{v}=\left(\mathrm{v}_{\mathrm{R}}+\mathrm{v}_{\mathrm{L}}\right) / 2 \\
& \mathrm{w}_{\mathrm{o}}=\left(\mathrm{v}_{\mathrm{R}}-\mathrm{v}_{\mathrm{L}}\right) / 2 \mathrm{~d} \\
& \mathrm{w}_{\mathrm{R}}=\left(\mathrm{v}+\mathrm{dw}_{\mathrm{o}}\right) / \mathrm{r}
\end{aligned}
$$

$\mathrm{w}_{\mathrm{L}}=\left(\mathrm{v}-\mathrm{dw}_{\mathrm{o}}\right) / \mathrm{r}$ 


$$
\begin{aligned}
& \frac{\mathrm{d} \psi}{\mathrm{dt}}=\mathrm{w}_{\mathrm{o}} \\
& \frac{\mathrm{dX}}{\mathrm{dt}}=\mathrm{v} \cdot \cos (\psi) \\
& \frac{\mathrm{dY}}{\mathrm{dt}}=\mathrm{v} \cdot \sin (\psi)
\end{aligned}
$$

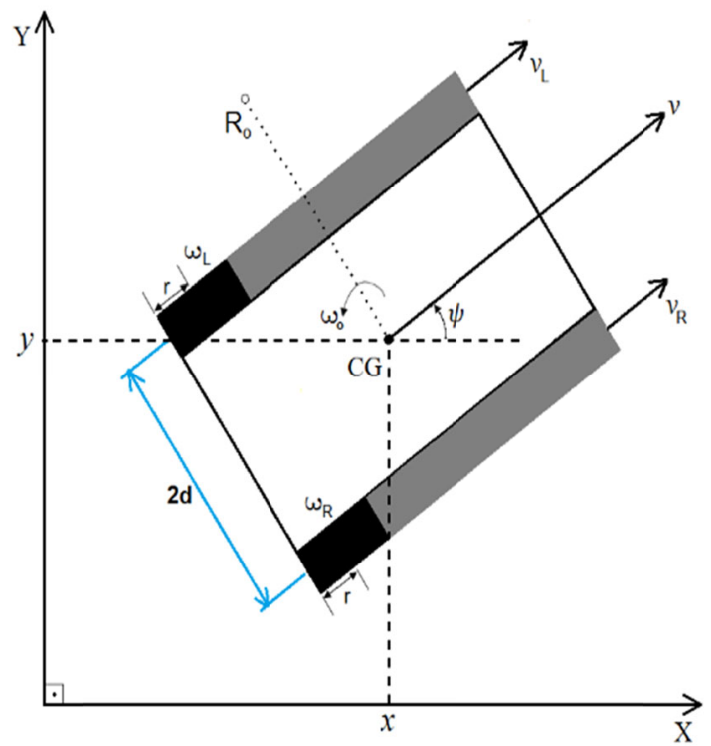

Şekil 3. Kinematik model

Burada; $r$ palet tahrik dişli yarıçapı, $\mathrm{w}_{\mathrm{R}}, \mathrm{w}_{\mathrm{L}}$ sağ ve sol palet tahrik dişli açısal hızı, $\mathrm{w}_{\mathrm{o}}$ paletli aracın açısal hızı, $\mathrm{v}_{\mathrm{R}}, \mathrm{v}_{\mathrm{L}}$ sağ ve sol palet hızı, v paletli aracın hızı, $\psi$ paletli aracın $\mathrm{x}$ eksenine göre açısıdır.

\subsubsection{Dinamik Model}

Paletli aracın dinamik modeli, Şekil 4'de gösterilen koordinat düzlemindeki görünüşünden faydalanılarak çıkarılmış ve matematiksel ifadeler Eşitlik 9-20'de verilmiştir [13,14].

$$
\mathrm{ma}=\mathrm{F}_{\mathrm{T}}-\mathrm{F}_{\mathrm{S}}
$$

$\mathrm{m} \frac{\mathrm{dv}}{\mathrm{dt}}=\mathrm{F}_{\mathrm{R}}+\mathrm{F}_{\mathrm{L}}-\mathrm{F}_{\mathrm{S}}$

$(6)$

$$
\mathrm{J} \varepsilon=\mathrm{M}_{\mathrm{T}}-\mathrm{M}_{\mathrm{S}}
$$$$
\mathrm{J} \frac{\mathrm{dw}_{\mathrm{o}}}{\mathrm{dt}}=\left(\mathrm{F}_{\mathrm{R}}-\mathrm{F}_{\mathrm{L}}\right) \mathrm{d}-\mathrm{M}_{\mathrm{S}}
$$

$$
\mathrm{F}_{\mathrm{R}}=\frac{\mathrm{M}_{\mathrm{R}}}{\mathrm{r}}
$$

$$
\mathrm{M}_{\mathrm{R}}=\mathrm{N}_{\mathrm{g}} \mathrm{M}_{\mathrm{mR}}
$$

$$
\mathrm{M}_{\mathrm{mR}}=\mathrm{K}_{\mathrm{T}} \mathrm{I}_{\mathrm{r}}
$$

$$
\mathrm{F}_{\mathrm{R}}=\frac{\mathrm{N}_{\mathrm{g}} \mathrm{K}_{\mathrm{T}}}{\mathrm{r}} \mathrm{I}_{\mathrm{r}}
$$

$\frac{\mathrm{dv}}{\mathrm{dt}}=\frac{\mathrm{N}_{\mathrm{g}} \mathrm{K}_{\mathrm{t}}}{\mathrm{mr}} \mathrm{I}_{1}+\frac{\mathrm{N}_{\mathrm{g}} \mathrm{K}_{\mathrm{t}}}{\mathrm{mr}} \mathrm{I}_{\mathrm{r}}-\frac{1}{\mathrm{~m}} \mathrm{~F}_{\mathrm{s}}$

$\frac{\mathrm{dw}_{\mathrm{o}}}{\mathrm{dt}}=-\frac{\mathrm{N}_{\mathrm{g}} \mathrm{K}_{\mathrm{t}} \mathrm{d}}{\mathrm{Jr}} \mathrm{I}_{1}+\frac{\mathrm{N}_{\mathrm{g}} \mathrm{K}_{\mathrm{t}} \mathrm{d}}{\mathrm{Jr}} \mathrm{I}_{\mathrm{r}}-\frac{1}{\mathrm{~J}} \mathrm{M}_{\mathrm{s}}$

$\frac{\mathrm{dI}_{1}}{\mathrm{dt}}=-\frac{\mathrm{K}_{\mathrm{b}} \mathrm{N}_{\mathrm{g}}}{\mathrm{L}_{\mathrm{a}} \mathrm{r}} \mathrm{v}+\frac{\mathrm{K}_{\mathrm{b}} \mathrm{N}_{\mathrm{g}} \mathrm{d}}{\mathrm{L}_{\mathrm{a}} \mathrm{r}} \mathrm{w}_{\mathrm{o}}-\frac{\mathrm{R}_{\mathrm{a}}}{\mathrm{L}_{\mathrm{a}}} \mathrm{I}_{1}+\frac{1}{\mathrm{~L}_{\mathrm{a}}} \mathrm{U}_{1}$

$\frac{\mathrm{dI}_{\mathrm{r}}}{\mathrm{dt}}=-\frac{\mathrm{K}_{\mathrm{b}} \mathrm{N}_{\mathrm{g}}}{\mathrm{L}_{\mathrm{a}} \mathrm{r}} \mathrm{v}-\frac{\mathrm{K}_{\mathrm{b}} \mathrm{N}_{\mathrm{g}} \mathrm{d}}{\mathrm{L}_{\mathrm{a}} \mathrm{r}} \mathrm{w}_{\mathrm{o}}-\frac{\mathrm{R}_{\mathrm{a}}}{\mathrm{L}_{\mathrm{a}}} \mathrm{I}_{\mathrm{r}}+\frac{1}{\mathrm{~L}_{\mathrm{a}}} \mathrm{U}_{\mathrm{r}}$

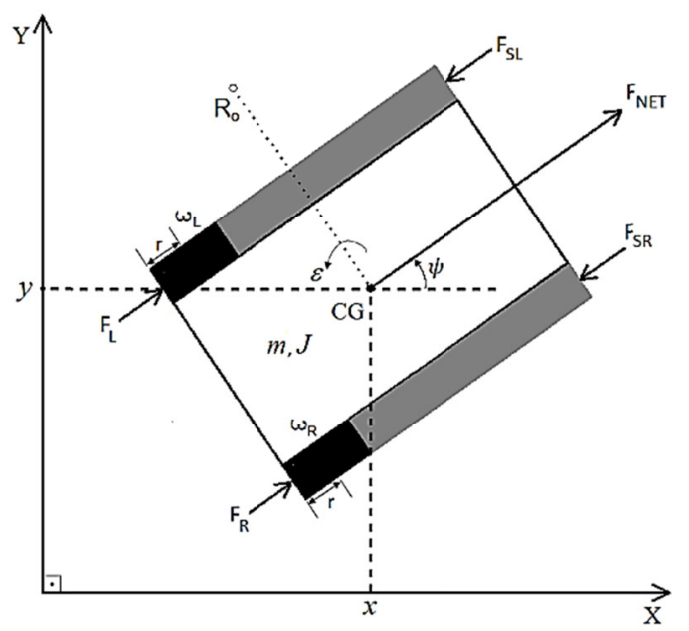

Şekil 4. Dinamik model 
Burada; m,a paletli aracın kütlesi ve ivmesi, $\mathrm{F}_{\mathrm{R}}, \mathrm{F}_{\mathrm{L}}, \mathrm{F}_{\mathrm{T}}, \mathrm{F}_{\mathrm{S}}$ sağ, sol, toplam ve sürtünme kuvvetleri, J,c paletli aracin eylemsizlik momenti ve açısal dönme ivmesi, $\mathrm{M}_{\mathrm{T}}, \mathrm{M}_{\mathrm{S}}$ toplam ve sürtünme momentleri, $\mathrm{N}_{\mathrm{g}}$ dişli dönüştürme oran1, $\mathrm{d}$ paletler arası mesafe, $\mathrm{K}_{\mathrm{T}} \mathrm{DA}$ motor tork sabiti, $\mathrm{I}_{\mathrm{r}}, \mathrm{I}_{1}$ sağ ve sol motor akımıdır.

\section{YÖNTEM}

\subsection{Matlab/Simulink Modelinin Oluşturulması}

Aracın Matlab/Simulink modeli Şekil 5'de gösterilmiştir. Aracın kinematik modeli Eşitlik 4-8'den faydalanılarak, dinamik modeli ise Eşitlik 17-20'den faydalanılarak Şekil 6'daki gibi oluşturulmuştur.

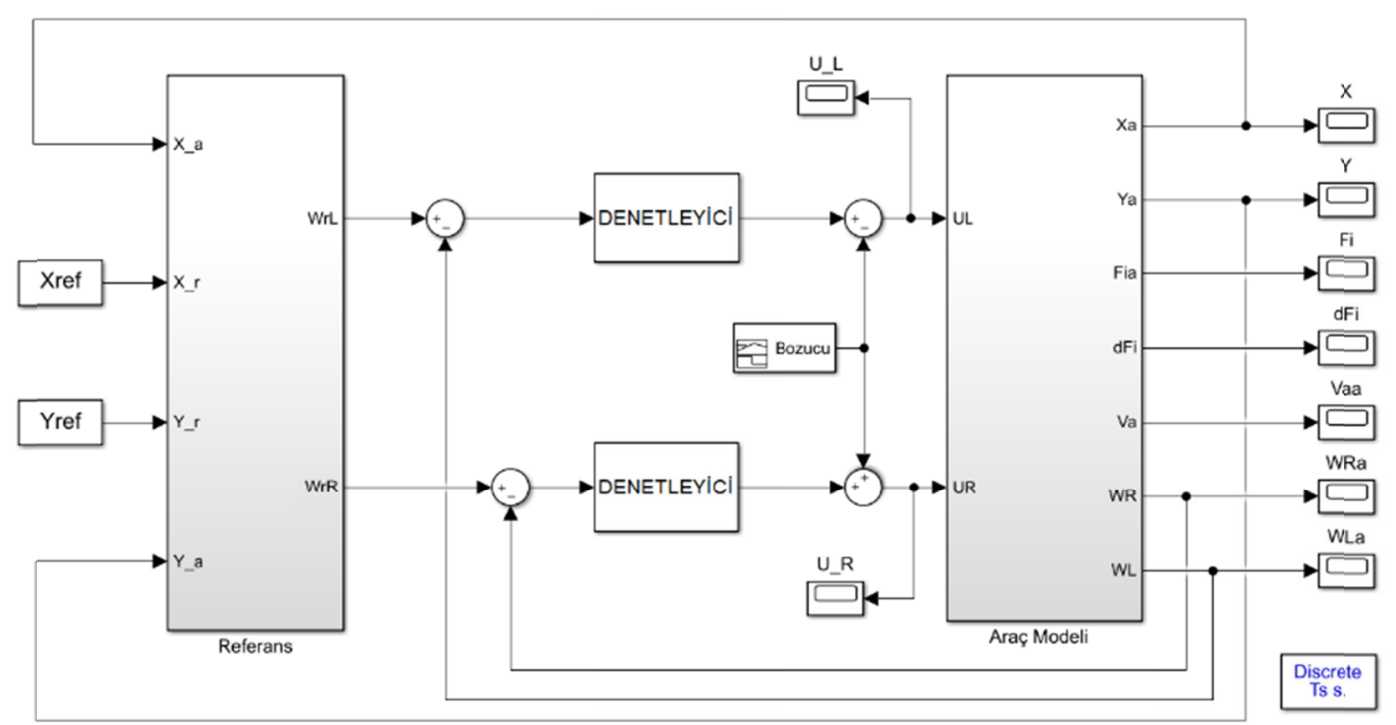

a)

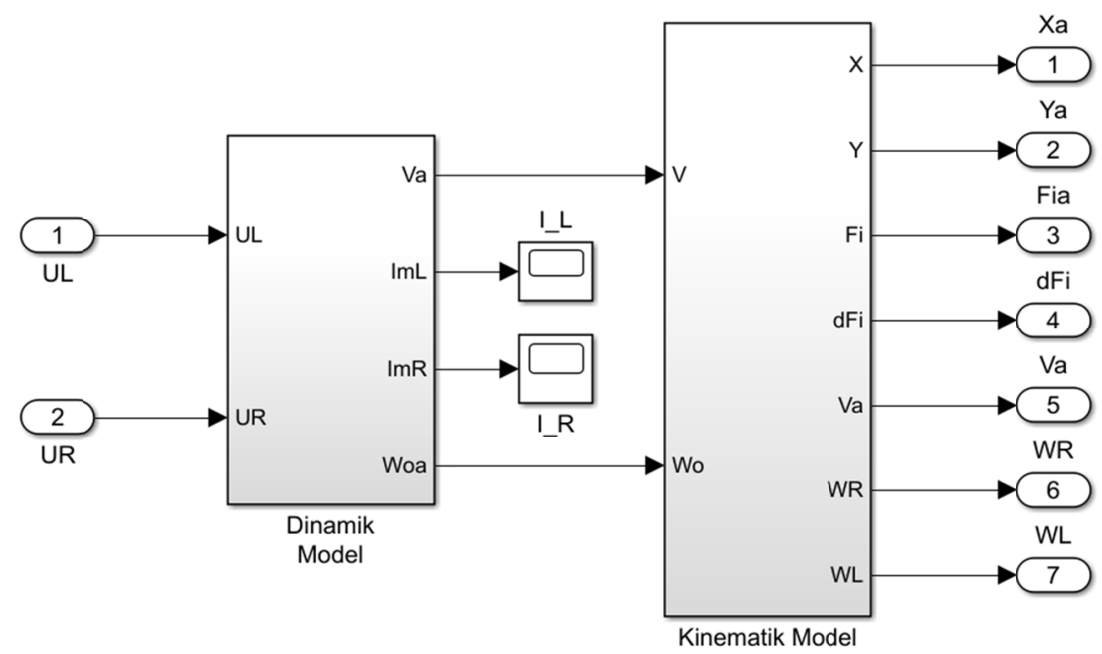

b)

Şekil 5. Sistemin matlab/simulink blok diyagramı (a), Araç modeli iç yapısı (b) 


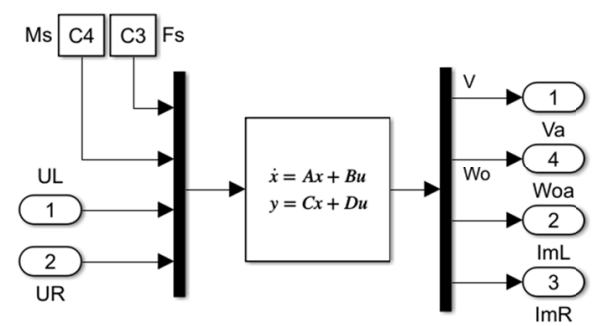

a)

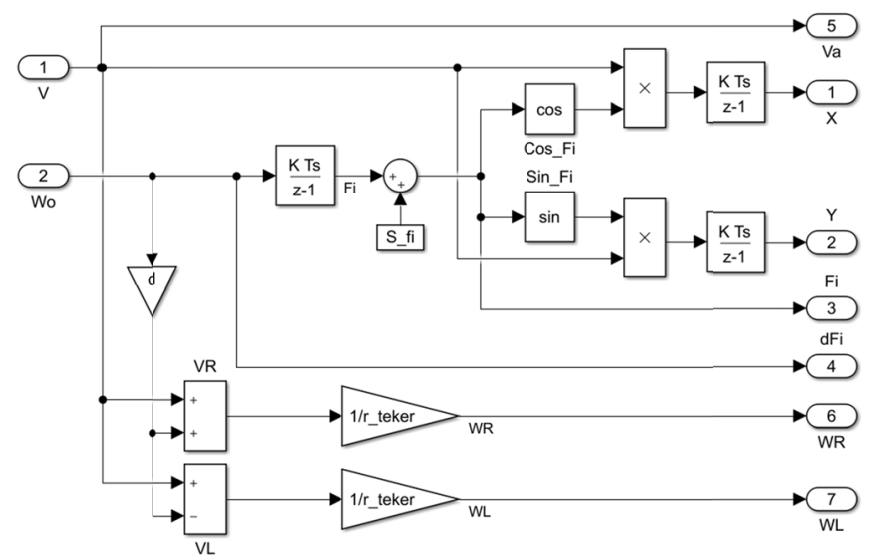

b)

Şekil 6. Paletli aracın dinamik modeli (a) ve paletli aracın kinematik modeli (b)

Şekil 6a'daki modelin durum uzay denklemleri Eşitlik 21-28'de verilmiştir.

$$
\begin{aligned}
& X=\left[\begin{array}{llll}
v & w_{0} & I_{L} & I_{R}
\end{array}\right]^{T} \\
& U=\left[\begin{array}{llll}
F_{S} & M_{S} & U_{L} & U_{R}
\end{array}\right]^{T} \\
& \dot{X}=A X+B U \\
& Y=C X+D U
\end{aligned}
$$

$$
A=\left[\begin{array}{cccc}
0 & 0 & \frac{\mathrm{N}_{g} K_{t}}{m r} & \frac{N_{g} K_{t}}{m r} \\
0 & 0 & -\frac{N_{g} K_{t} d}{J r} & \frac{N_{g} K_{t} d}{J r} \\
-\frac{K_{b} N_{g}}{L_{a} r} & \frac{K_{b} N_{g} d}{L_{a} r} & -\frac{R_{a}}{L_{a}} & 0 \\
-\frac{K_{b} N_{g}}{L_{a} r} & -\frac{K_{b} N_{g} d}{L_{a} r} & 0 & -\frac{R_{a}}{L_{a}}
\end{array}\right]
$$

$$
\mathrm{B}=\left[\begin{array}{cccc}
-\frac{1}{\mathrm{~m}} & 0 & 0 & 0 \\
0 & -\frac{1}{\mathrm{~J}} & 0 & 0 \\
0 & 0 & \frac{1}{\mathrm{~L}_{\mathrm{a}}} & 0 \\
0 & 0 & 0 & \frac{1}{\mathrm{~L}_{\mathrm{a}}}
\end{array}\right]
$$$$
\mathrm{C}=\left[\begin{array}{llll}
1 & 0 & 0 & 0 \\
0 & 1 & 0 & 0 \\
0 & 0 & 1 & 0 \\
0 & 0 & 0 & 1
\end{array}\right]
$$ 


\subsection{Denetleyici Tasarım}

Denetleyicilere giriş olarak sağ ve sol tekerlerin açısal hız hataları verilmiştir. Denetleyici çıkışı Eşitlik 22'deki $U$ matrisinin $U_{L}$ ve $U_{R}$ kontrol işaretlerini oluşturmaktadır. $\mathrm{Bu}$ işaretler $\mathrm{DA}$ motorlarına verilen gerilimlerdir.

\subsubsection{PI Tipi Denetleyicinin Tasarımı}

Aracın birim basamak tepkisini elde etmek için motorlara sabit $24 \mathrm{~V}$ gerilim verilerek, maksimum hız olan $1,15 \mathrm{~m} / \mathrm{s}^{\prime}$ ye ulaşması sağlanmıştır. Şekil 7'deki açık-çevrim grafik elde edilmiştir. Paletli aracın açık-çevrim birim basamak tepkisine göre, $\mathrm{D}=0,067 \mathrm{~s}$ ve $\mathrm{T}=0,753 \mathrm{~s}$ olarak ölçülmüştür.

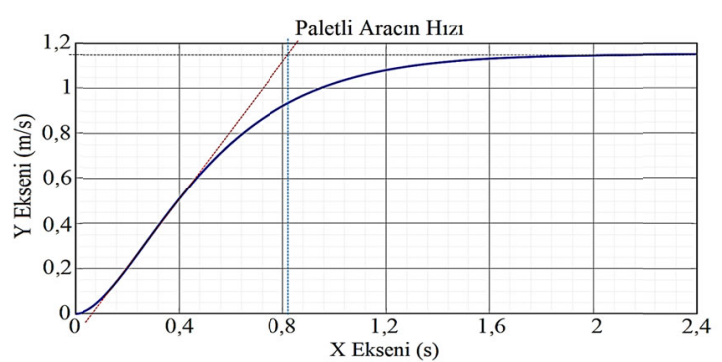

Şekil 7. Araç hızının açık-çevrim birim basamak tepkisi

$\mathrm{Bu}$ değerlere göre Ziegler-Nichols yöntemiyle hesaplanan PID parametreleri Çizelge 3'de verilmiştir [15]. PI denetleyicisinin parametreleri $\mathrm{K}_{\mathrm{p}}=10,115$ ve $\mathrm{T}_{\mathrm{i}}=0,223$ olarak hesaplanmıștır.

Çizelge 3. Paletli aracın açık çevrim zieglernichols PID parametreleri

\begin{tabular}{|c|c|c|c|}
\hline Denetleyici Türü & $\mathrm{K}_{\mathrm{p}}$ & $\mathrm{T}_{\mathrm{i}}$ & $\mathrm{T}_{\mathrm{d}}$ \\
\hline $\mathrm{P}$ & 11,239 & $\infty$ & 0 \\
\hline PI & 10,115 & 0,223 & 0 \\
\hline PID & 13,487 & 0,134 & 0,034 \\
\hline
\end{tabular}

\subsubsection{Bulanık Mantık Denetleyicinin Tasarımı}

Bulanıklaştırma, bir sistemden alınan net bir giriş veri kümesinin bulanık dilsel değişkenler, bulanık dilsel terimler ve üyelik fonksiyonları kullanılarak bulanık bir kümeye dönüştürülmesidir. Çıkarım birimi, bulanık giriş setine kural tabanındaki kuralları uygulayarak bir bulanık çıkış seti oluşturur. Bulanık çıkarım yöntemlerinde en yaygın olan ve bu çalışmada kullanılan yöntem Mamdani yöntemidir. Bulanıklaştırma, sonuçtaki bulanık çıktı üyelik işlevleri kullanılarak net bir çıktıya dönüştürülür. Sonuç olarak, bu işlem uygulamada kullanılacak gerçek değerleri sağlar $[16,17]$

Şekil 8'de bir Bulanık Mantık Sistemi (BMS) blok diyagramı verilmiştir. Bu çalışmadaki BMD iki giriş (hata (e) ve hata değişimi $(\Delta \mathrm{e})$ ) ve bir çıkış (motor besleme gerilimi (U))' tan oluşmaktadır.

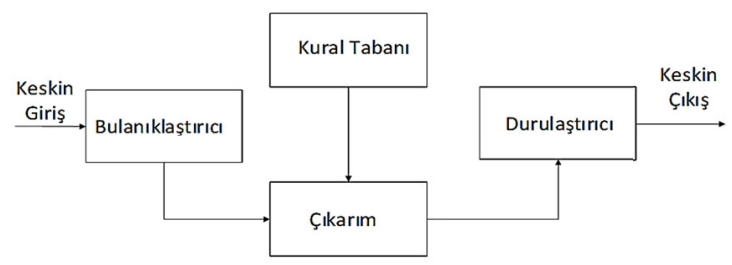

Şekil 8. Bulanık mantık sistemi blok diyagramı

Sürekli değişkenlerde, herhangi bir aralığı temsil eden bulanık kümelerin sayısı üç ile yedi arasında seçilebilir. Genellikle üç sayısının hassasiyeti az olması ve yedi sayısının da hesaplama yükü getirmesinden dolayı makul olarak beş sayısı seçilmektedir. Gauss tipi üyelik fonksiyonu diğer fonksiyon tiplerine göre daha az parametre gerektirmesi sayesinde birçok çalışmada tercih edilmektedir [18]. Bu çalışmada giriş ve çıkışlar için Şekil 9'daki gibi 5'er Gauss üyelik fonksiyonu NB (Negatif Büyük), NK (Negatif Küçük), S (Sıfır), PK (Pozitif Küçük) ve PB (Pozitif Büyük) olarak seçilmiş ve Çizelge 4'de verilen toplam 25 kural ile tanımlanmıştır. Durulaştırma yöntemi olarak ağırlık merkezi yöntemi kullanılmıștır [19].

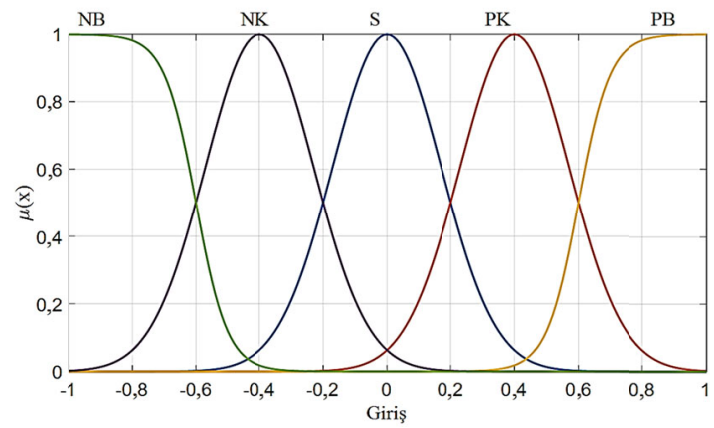

Şekil 9. Bulanık üyelik fonksiyionları 
Çizelge 4. Bulanık mantık kural tablosu

\begin{tabular}{|c|c|c|c|c|c|c|}
\hline \multicolumn{2}{|c|}{ U } & \multicolumn{5}{|c|}{$\Delta \mathrm{e}$} \\
\cline { 3 - 7 } & NB & NK & S & PK & PB \\
\hline \multirow{4}{*}{ e } & NB & NB & NB & NB & NK & S \\
\cline { 2 - 7 } & NK & NB & NB & NK & S & PK \\
\cline { 2 - 7 } & S & NB & NK & S & PK & PB \\
\cline { 2 - 7 } & PK & NK & S & PK & PB & PB \\
\cline { 2 - 7 } & PB & S & PK & PB & PB & PB \\
\hline
\end{tabular}

\section{BENZETIMM ÇALIŞMALARI}

Geliştirilen araç modeli için oluşturulan denetleyicilerin sinüs işaretli bir referans yörüngeyi takip etme başarısı Şekil 10'da gösterilmiştir.

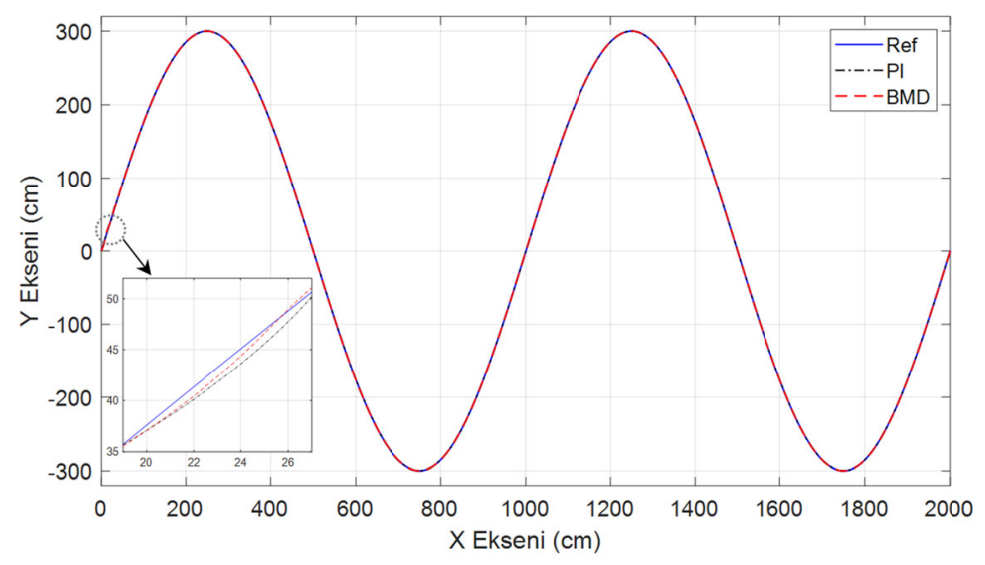

Şekil 10. Referans ve araç yörüngeleri

Her iki denetleyicinin çalışmasında hata çok küçük olduğundan, denetim sonuçlarını daha iyi görüntülemek amacıyla çalışmanın 21-27 cm aralığı büyütülerek şekil üzerinde gösterilmiştir. Denetleyicilerin bozucu girişlere karşı performansını ölçebilmek amacıyla aracın sağ ve sol motorlarına 43. saniyede 12 V'luk bir bozucu gerilim 1 saniye süre ile uygulanmış ve 44 . saniyede kaldırılmıştır. Bu çalışma durumunda elde edilen yörünge, kontrol işaretleri ve hata grafikleri Şekil 10-13'de verilmiştir.

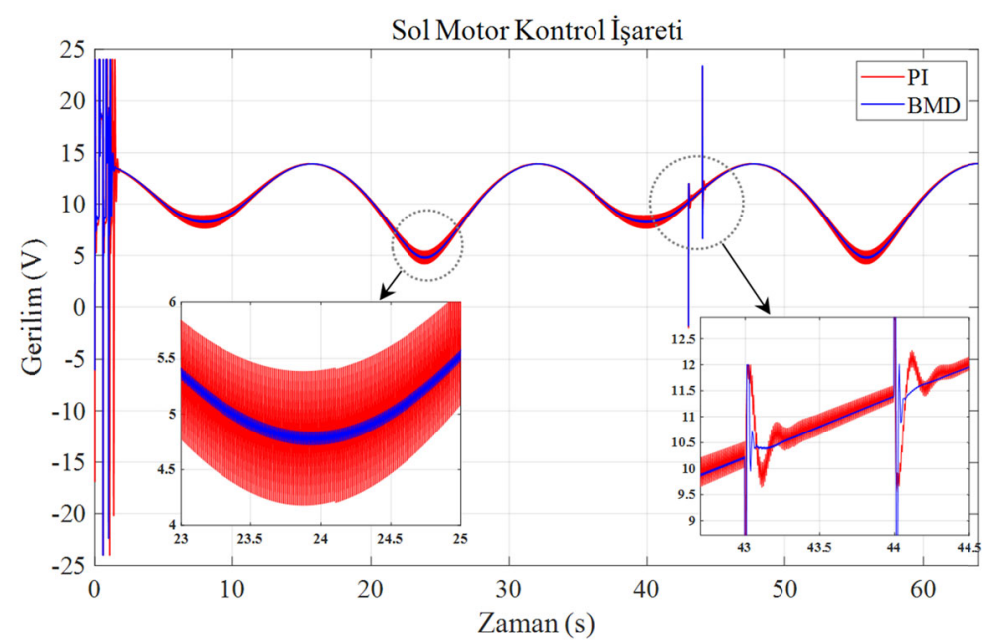

Şekil 11. Sol motor denetleyici çıkış işaretleri 


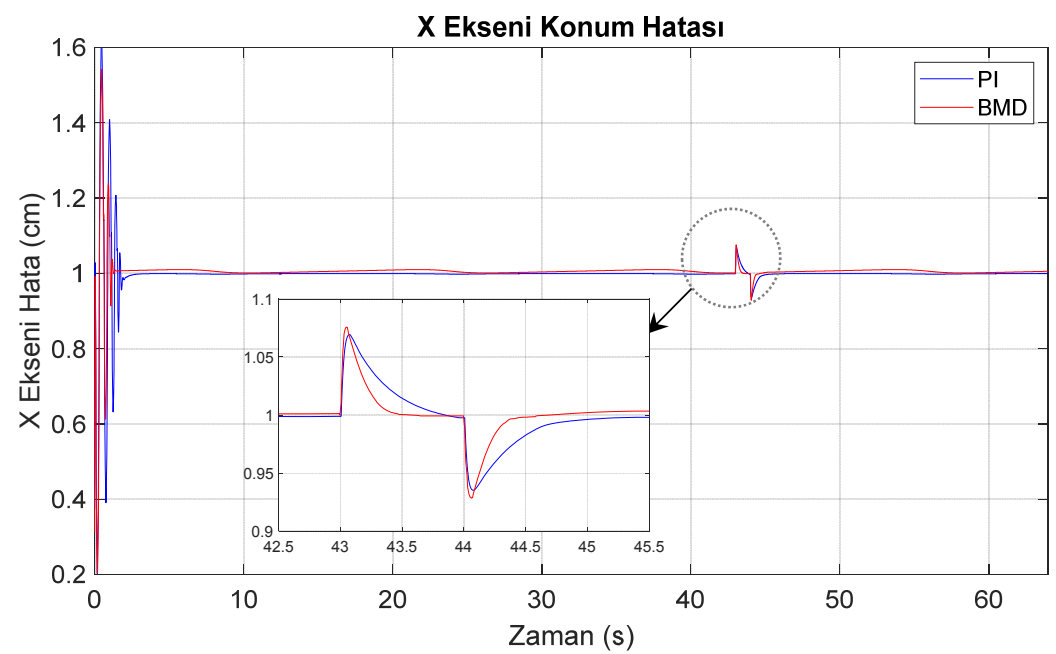

Şekil 12. Araç X-ekseni konum hataları

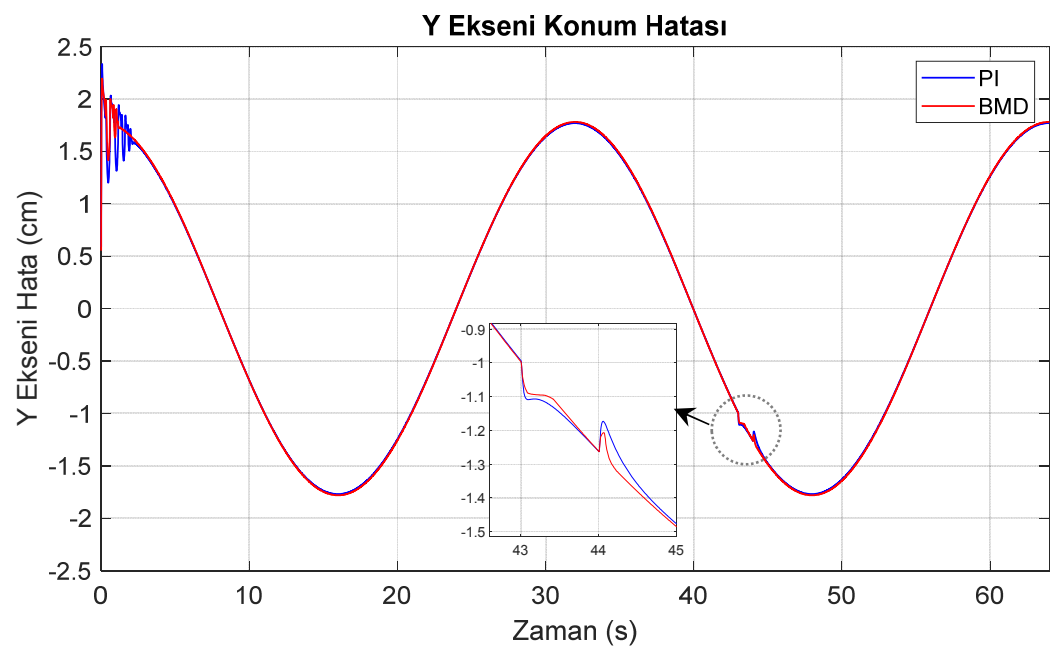

Şekil 13. Araç y-ekseni konum hataları

\section{TARTIŞMA VE SONUÇLAR}

$\mathrm{Bu}$ çalışmada gerçek bir paletli otonom aracın sistem modeli oluşturularak bulanık mantık denetleyici ile yörünge kontrolü gerçekleştirilmiştir. Kullanılan denetleyicinin performansı klasik PI denetleyici ile karşılaştırılmıştır.

Şekil 10'daki yörüngeler incelendiğinde, başlangıç anında BMD'nin, PI denetleyiciden daha kısa sürede kararlı hale geçtiği görülmüştür.
Şekil 11'deki sol motora uygulanan kontrol sinyali incelendiğinde PI denetleyici en fazla 1,2 V, BMD ise en fazla 0,1 V'luk bir salınım yaptığ görülmektedir. Sisteme verilen bozucu girişin etkisini PI denetleyici 0,3 s'de düzeltirken, BMD ise $0,2 \mathrm{~s}$ 'de düzeltmiştir.

Şekil 12'deki x-ekseni konum hata grafiği incelendiğinde her iki denetleyicinin $1 \mathrm{~cm}$ hata ile sistemi takip ettiği görülmektedir. Bu hata değeri, referans sinyal ile araç yörüngesi arasındaki hata olmayıp aracın referans sinyali zaman olarak geriden takip etmesinden kaynaklanmaktadır. 
Sisteme bozucu giriş uygulandığında meydana gelen yörünge sapmasını PI denetleyici 0,8 s'de düzeltirken, BMD 0,5 s'de düzeltmiştir.

Şekil 13'de ise y-ekseni konum hata grafiği incelendiğinde, her iki denetleyicinin referans sinyali $\pm 1,8 \mathrm{~cm}$ arasında değişen bir hata ile takip ettiği görülmektedir. Bozucu girişin sistemde oluşturduğu etkiyi BMD, PI denetleyiciden daha kısa sürede ortadan kaldırmıştır.

Tüm çalışma durumları incelendiğinde BMD'nin PI denetleyiciye göre daha başarılı olduğu açıkça görülmektedir. Karmaşık bir modele sahip olan paletli araçların yörünge kontrolünde BMD tipi denetleyicinin simülasyon ortamında başarı olduğu görülmüş̧ür. Bulanık mantık esaslı denetleyicilerin paletli otonom araçların gerçek zamanlı uygulamalarında kullanılabileceği öngörülmektedir.

\section{TEŞEKKÜR}

Yazarlar, bu çalışmanın yapımında kendilerinden desteklerini esirgemeyen mesai arkadaşlarına teşekkür eder.

\section{KAYNAKLAR}

1. Dong, H., Luo, Z., 2011. Control Strategies of Human Interactive Robot Under Uncertain Environments. Mobile Robots: Control Architectures, Bio-Interfacing, Navigation, Multi Robot Motion Planning and Operator Training ( $2^{\text {nd }}$ Edition). Intech, Croatia, p. 390. https://doi.org/10.5772/2304.

2. Blasko, V., Kaura, V., 1997. A New Mathematical Model and Control of a Threephase AC-DC Voltage Source Converter. IEEE Transactions on Power Electronics, 12(1), 116-123.

3. Kececioglu, F.O., Acikgoz, H., Yildiz, C., Gani, A., Sekkeli, M., 2017. Power Quality Improvement Using Hybrid Passive Filter Configuration for Wind Energy Systems. Journal of Electrical Engineering and Technology, 12(1), 207-216.
4. Eltamaly, A.M., Alolah, A.I., Badr, B.M., 2010. Fuzzy Controller for Three Phases Induction Motor Drives. IEEE 2010 International Conference on Autonomous and Intelligent Systems, 1-6.

5. Kılıç, E., Özçalık, H.R., Şit, S., 2018. Adaptive Controller with RBF Neural Network for Induction Motor Drive. International Journal of Numerical Modelling. Electronic Networks, Devices and Fields, 31(3), 1-11.

6. Şit, S., Özçalik, H.R., Kılıç, E., Doğmuş, O., 2016. Asenkron Motorların Online Adaptif Sinirsel-Bulanık Denetim (ANFIS) Sistemine Dayalı Hız Denetim Performansının İncelenmesi. Çukurova Üniversitesi Mühendislik Mimarlık Fakültesi Dergisi, 31(ÖS 2), 33-41.

7. Sidi, M.H.A., Hudha, K., Kadir, Z.A., Amer, N.H., 2018. Modeling and Path Tracking Control of a Tracked Mobile Robot. 2018 IEEE 14th International Colloquium on Signal Processing and its Application, 9-10 March, 72-76.

8. Huang, P., Zhang, Z., Luo, X., Zhang, J., Huang, P., 2018. Path Tracking Control of a Differential-Drive Tracked Robot Based on Look-ahead Distance. IFAC-Papers On Line, 51(17), 112-117.

9. Asai, M., Chen, G., Takami, I., 2019. Neural Network Trajectory Tracking of Tracked Mobile Robot. $16^{\text {th }}$ International MultiConference on Systems, Signals and Devices, (4), 225-230.

10. Ji, P., Li, S., Xu, M., Li, J., Guo, J., 2018. Design of Sliding Cloud-Model Cross Coupling Controller for Tracked Mobile Robot. Proceedings of the $37^{\text {th }}$ Chinese Control Conference, July 25-27, 5353-5357.

11. Jayakumar, V., Kumar, M., 2012. Engineering Mechanics. New Delhi: PHI Learning Private Limited.

12. Malu, S.K., Majumdar, J., 2014. Kinematics, Localization and Control of Differential Drive Mobile Robot. Global Journal of Researches in Engineering: Robotics \& Nano-Tech, 14(1), 1-9.

13. Gholipour, A., Yazdanpanah, M.J., 2003. Dynamic Tracking Control of Nonholonomic Mobile Robot with Model Reference 
Adaptation for Uncertain Parameters. 2003 European Control Conference, 3118-3122.

14. Wu, X., Xu, M., Wang, L., 2013. Differential Speed Steering Control for Four-wheel Independent Driving Electric Vehicle. IEEE International Symposium on Industrial Electronics, 1(4), 355-359.

15. Ogata, K., 2010. Modern Control Engineering ( $5^{\text {th }}$ Edition). Prentice Hall, New Jersey, 905.

16. Kayacan, E., Khanesar, M.A., 2015. Fuzzy Neural Networks For Real Time Control Applications ( $1^{\text {st }}$ Edition). Butterworth Heinemann, Boston, 264. https://doi.org/10. 1016/C2014-0-02444-6

17. Antao, R., 2017. Type-2 Fuzzy Logic Uncertain Systems Modeling and Control. Higher Education Press, Beijing, 136. https://doi.org/ 10.1007/978-981-10-4633-9

18. Ocampo-duque, W., Osorio, C., Piamba, C., Schuhmacher, M., Domingo, J.L. 2013. Water Quality Analysis in Rivers with Nonparametric Probability Distributions and Fuzzy Inference Systems: Application to the Cauca River, Colombia. Environment International, 52, 17-28.

19. Aisbett, J., Rickard, J.T., 2014. Centroids of Type-1 and Type-2 Fuzzy Sets When Membership Functions Have Spikes. IEEE Transactions on Fuzzy Systems, 22(3), 685-692. 
